\title{
LA ECOPEDAGOGÍA EN CUESTIÓN: UNA REVISIÓN BIBLIOGRÁFICA
}

\author{
Soraya María Ruiz-Peñalver \\ Universidad de Cádiz \\ Laura Porcel-Rodríguez \\ Universidad de Granada \\ Ana Isabel Ruiz-Peñalver \\ Euroseper (Granada)
}

\begin{abstract}
RESUMEN: En una sociedad dinámica y cada vez más globalizada, los contextos educativos han de adaptarse a estas situaciones cambiantes. En este sentido se enmarca la ecopedagogía, una estrategia pedagógica crítica que trata de estimular una educación que incentive la concienciación ambiental, la equidad y la justicia social, fomentando el sentimiento de comunidad global. Con esta intención se desarrolló la educación para el desarrollo sostenible (EDS), impulsada por la UNESCO. Sin embargo, en un mundo marcado por el pensamiento neoliberal, esta finalidad se ha traspuesto en prácticas que no profundizan en los problemas planteados por la ecopedagogía, y que, por tanto, distan de estas premisas. A partir de una revisión sistemática de la literatura sobre ecopedagogía, el objetivo de este artículo es analizar qué se está investigando en este ámbito y cómo la ecopedagogía se readapta a las necesidades sociales, económicas y ambientales cambiantes. Los principales resultados muestran que la mayoría de las aportaciones tienen un marcado carácter teórico, y muchas de ellas son críticas con el actual contexto educativo. En términos generales, el ámbito académico considera necesaria la inclusión de importantes cambios en el sistema educativo que incluyan los principios originales de la ecopedagogía.
\end{abstract}

PALABRAS CLAVE: ecopedagogía, educación para el desarrollo sostenible, revisión bibliográfica.

\section{QUESTIONING ECOPEDAGOGY: A LITERATURE REVIEW}

ABSTRACT: In a dynamic and globalized society, educational contexts have to adapt to these changing situations. In this sense, ecopedagogy is a critical pedagogical strategy that stimulates an education that encourages environmental awareness, equity and social justice, fostering the feeling of 
global community. Education for Sustainable Development (ESD), promoted by UNESCO, was developed with this intention as well. However, in a neoliberal context, this aim is transposed into practices that do not delve into the problems posed by ecopedagogy, and therefore, are far from these premises. Based on a systematic review of the literature on eco-pedagogy, this paper aims to analyze how scholars approach this field and how ecopedagogy is readapted to social, economic and environmental needs. The main results show that most of the contributions are theoretical, and many of them are critical of the current educational context. In general terms, the academic field considers it necessary to include important changes in the educational system that include the original principles of ecopedagogy.

KEYWORDS: Ecopedagogy, education for sustainable development, literature review.

Recibido: 14/01/2021

Aceptado: 19/03/2021

Correspondencia: Soraya María Ruiz-Peñalver, Facultad de Ciencias Económicas y Empresariales, Universidad de Granada, Campus Universitario de Cartuja, 18071 Granada. Email: soraya.ruiz@uca.es

\section{INTRODUCCIÓN}

Nos encontramos en un mundo cada vez más diverso y complejo, donde el impacto de la actual cultura neoliberal', los patrones de producción y consumo, el poder, la prepotencia y preponderancia política de algunas naciones, etc., es cada vez más evidente en actual orden mundial. En efecto, el calentamiento global, la pérdida de biodiversidad, la ingente generación de residuos, los continuados conflictos bélicos, la creciente brecha entre ricos y pobres, el individualismo empedernido, o la pérdida de la riqueza cultural, son solo algunos ejemplos. Asimismo, estos problemas tienen mayor incidencia en los países más vulnerables, quienes ejercen un menor impacto en los ámbitos ambiental, social y económico (Evans y Reid, 2014).

Ante este contexto, según el concepto de sostenibilidad de Brundtland (CMMAD, 1987), estos cambios no solo deben ligarse al ámbito ambiental, sino que es un importante desafío que debe considerar la paz, así como la equidad y la justicia social, entre otros. En este sentido, se formuló la Agenda 2030 para el Desarrollo Sostenible (ONU, 2015), una propuesta no exenta de matices neoliberales, donde la educación se plantea como un recurso para afrontar los complejos desafíos a los que se enfrenta la comunidad mundial. Sin embargo, se requiere una educación que transforme la formación de la ciudadanía buscando la reflexión en cómo, en nuestros territorios, se

1. Es una teoría de la práctica de la política económica que propone que el bienestar humano puede mejorar a través de la maximización de las libertades empresariales dentro de un marco institucional caracterizado por los derechos derivados de la propiedad privada, la libertad individual y unos mercados libres de trabas (Evans y Reid, 2014: 75). 
están desarrollando las relaciones económicas, políticas, culturales, éticas, raciales, de género, y ambientales, resultado de los cambios acaecidos a nivel mundial (Whiting et al., 2018; Rodrigues, 2018). Además, es necesario analizar las consecuencias de dichos cambios para estimular una cultura que haga viable la coexistencia de todos en el planeta (Matthews, 2014).

Como respuesta a esta crisis global, han surgido diferentes iniciativas en el ámbito de la educación para el desarrollo sostenible, como así lo manifiestan los sucesivos informes de las diferentes comisiones celebradas internacionalmente (IUCN, 1980; WCED, 1987), destacando la Cumbre de la Tierra en Brasil (1992), la Conferencia Tesalónica (1997) o la Declaración de Johannesburgo (2002), donde se han gestado acciones como la educación ambiental, la ecoeducación, la ecorientación o la educación para el desarrollo sostenible (EDS), con un importante calado, en gran parte, por el impulso motivado por la UNESCO, aunque no exentas de cierta ambigüedad conceptual (Ruiz et al., 2018). La EDS se formuló como una educación "que empodere a los individuos para reflexionar sobre sus propias acciones, tomando en cuenta sus efectos sociales, culturales, económicos y ambientales [...] para actuar en situaciones complejas de una manera sostenible". Para su implantación, la EDS requiere una "pedagogía transformadora y orientada a la acción, caracterizada por el aprendizaje autodidacta, la participación y la colaboración, la orientación hacia los problemas, la inter y transdisciplinariedad y la creación de vínculos entre el aprendizaje formal e informal" (UNESCO 2017: 7). Sin embargo, el virtuosismo de la EDS se ha traspuesto en prácticas que distan demasiado de esta finalidad (Evans y Reid, 2014; LæssØe, 2010), debido a la influencia del neoliberalismo. Para que el original concepto de sostenibilidad pueda llevarse a cabo, es necesaria la transformación del paradigma educativo, que requiere provocar reflexiones y discusiones pedagógicas en todos los ámbitos (Matthews, 2014).

En este sentido, la ecopedagogía se plantea como una propuesta muy adecuada para llevar a cabo la tan necesaria transformación educativa a nivel global. La ecopedagogía tiene sus orígenes en la década de los 70 del siglo XX con los trabajos de Paulo Freire (1921-1997), notable pedagogo brasileño considerado como el padre de la ecopedagogía crítica. Freire dio una orientación a la pedagogía hacia el respeto por el medio ambiente, argumentando la necesidad de educar a las nuevas generaciones en un paradigma de desarrollo sostenible, en el que las personas han de hacerse responsables de sus actos en la Tierra (Freire, 1993). Freire se aleja de la pedagogía como metodología de enseñanza antropocéntrica y la enfoca al entorno, para concienciar a una ciudadanía planetaria. Para él, el sujeto educativo no es sólo el docente y los educandos, sino la estructura indisoluble configurada por los educandos, los educadores y la Tierra (Freire, 1993). La visión pedagógica de Freire pretendía dar solución a la crisis educativa caracterizada actualmente por el fracaso y el abandono escolar, la evaluación basada en la memorización, el no estímulo de la creatividad ni la curiosidad, la no personalización del aprendizaje, etc. Según Freire, las raíces de la ecopedagogía se fundamentan en una educación problematizadora que se cuestiona por la visión antropocéntrica del aprendizaje y que pretende aportar un aprendizaje crítico, tomando como referencia la vida cotidiana y el entorno en el que se desarrolla. Se trata de una pedagogía donde lo importante es el sentir, no el saber sobre el medio ambiente, de una pedagogía informal democrática y para la vida cotidiana 
que se centra en la vida, ya que incluye a todas las personas y culturas, además de respetar la diversidad (Magalhaes, 2013; Gutiérrez y Prado, 2004).

Además de las aportaciones de Freire, la ecopedagogía o pedagogía de la Tierra se dio a conocer globalmente, en el Foro Global Mundial de 1992, presentándose como un movimiento social y político (Fernández y Conde, 2010). Dicho movimiento surgió a raíz de la demanda social, organizaciones, ecologistas, educadores y trabajadores preocupados por cuestiones sociales, económicas, políticas, medioambientales, etc., y la firma de la Carta de la Tierra (CT) de 1997 fue el caldo de cultivo para su desarrollo. La ecopedagogía ha sido respaldada por autores como Gadotti (2000), Gutiérrez y Prado (2004) y Antunes y Gadotti (2006), aunque también hay que destacar los trabajos de Leonardo Boff, Fritjof Capra, Sebastiao Salgado, Boaventura de Sousa Santos y Milton Santos, que respaldaron los trabajos de Freire, apoyándose no sólo en la educación, sino en cómo la ecopedagogía ofrece un conjunto de conocimientos y valores más allá de la propia educación. Tamarit y Sánchez (2004: 3) indican que estos valores se sintetizan en:

- Diversidad e interdependencia de la vida.

- Preocupación común de la humanidad por vivir con todos los seres del planeta.

- Respeto a los Derechos Humanos.

- Desarrollo sostenible.

- Ética, Justicia, Equidad y comunidad.

- Prevención de lo que puede causar daño.

Por lo tanto, la ecopedagogía se vincula a un proyecto de futuro que modifica las dimensiones territoriales, éticas, políticas y relacionales (Huckle y Wals, 2015). Es, por tanto, un movimiento que va más allá de la pedagogía tradicional y que cuenta con una vocación crítica y transformadora, que Antunes y Gadotti (2006) han definido como el movimiento social y político complejo, que pretende la reorientación curricular y la consideración de una pedagogía diferente y crítica a otras pedagogías, cuyo reto es ensayar la vivencia de un nuevo paradigma: la visualización del planeta como una única comunidad.

Debido a la importancia que tiene la ecopedagogía en un contexto de crisis, y a su marcado carácter transversal y multidisciplinar, el objetivo de este documento es realizar una revisión sistemática de la literatura sobre ecopedagogía para analizar el estado de la cuestión, la evolución de la investigación en el tema y sus tendencias desde el ámbito académico.

\section{Método}

Una revisión sistemática supone un medio de identificación, evaluación e interpretación de todas las búsquedas disponibles relevantes referidas a un tema o a algún fenómeno de interés (Kitcharoen, 2004), en nuestro caso la ecopedagogía. Este análisis desarrolla un proceso transparente y reproducible de selección y estudio de investigaciones, implicando un enfoque cuantitativo y cualitativo (Denyer y Tranfield, 2009). 
Figura 1. Esquema metodológico

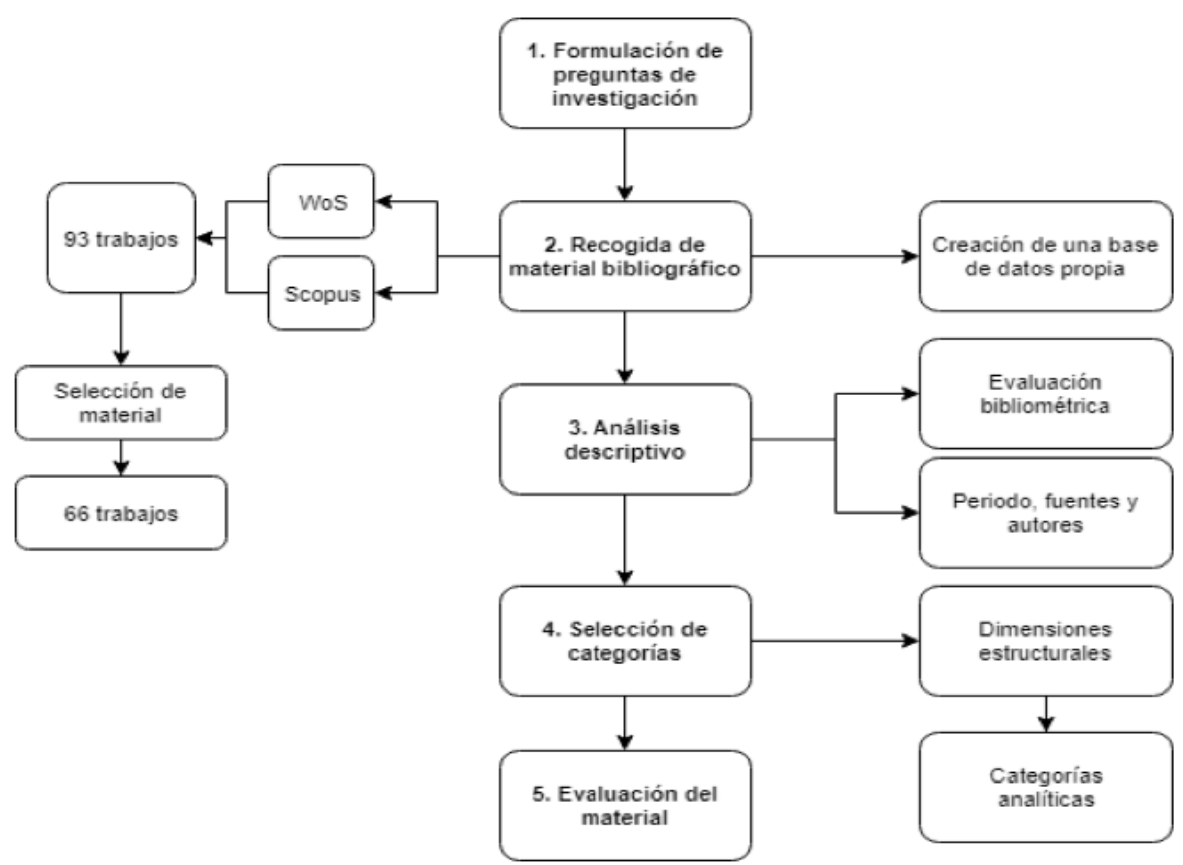

Tras considerar la importancia de la ecopedagogía (etapa 1), se procedió con la búsqueda de bibliografía (etapa 2). Para ello se consultaron Web of Science (WoS) y Scopus, las bases de datos de carácter científico más completas y rigurosas (Aghaei et al., 2013), ideales para implementar estudios bibliométricos fiables (Durán et al., 2016). La búsqueda se complementó con aportaciones seminales que no aparecían en estas bases de datos. Dicha búsqueda se inició introduciendo como palabras clave ecopedagogy y ecopedagogía. En el caso de Scopus, se emplearon los criterios de búsqueda Título, Autor, Palabras Clave, Resumen, mientras que, para WoS, se seleccionó la Colección principal de Web of Science, empleándose los mismos criterios. La exploración se realizó en noviembre de 2019 y no se aplicaron limitaciones de carácter cronológico ni de otro tipo. Tras estas búsquedas se creó una base de datos con todas las aportaciones encontradas, y se eliminó el material repetido.

La tercera fase se centró en un análisis descriptivo de la literatura, que conlleva un estudio tanto cuantitativo, que analiza las bases de datos consultadas en materia de ecopedagogía, como cualitativo, que analiza la evolución de las publicaciones, fuentes, etc., seleccionadas. Para ello, se aplicaron los siguientes índices:

El Índice de Meyer (Meyer Index, Relative Index of Uniqueness) representa la capacidad de la base de datos de cubrir un tema específico. A mayor valor, mayor es la cobertura que hace esa base de datos (Durán et al., 2016).

$$
\text { Índice Meyer }=\frac{\sum \text { Artículos duplicados } \times \text { peso específico }}{\text { Total de artículos }}
$$


Gluck (1999) elaboró un índice para medir el solapamiento entre varias bases de datos (Tradicional Overlap), y se emplea para estimar su nivel de similitud. Cuanto mayor es el valor obtenido, mayor es la similitud entre ambas bases de datos.

$$
\text { Índice de Solapamiento }=100 \times\left(\frac{\mid \text { Scopus } \cap \text { WoS } \mid}{\mid \text { Scopus } \cup W o S \mid}\right)
$$

Por último, se aplicaron dos índices para calcular el solapamiento de una base de datos sobre otra, teniendo en cuenta el peso del material que se encuentra en ambas bases de datos respecto de los de presencia única.

$$
\begin{gathered}
\% \text { Duplicados relativos Scopus }=100 \times\left(\frac{\mid \text { Scopus } \cap \text { WoS } \mid}{\mid \text { Scopus } \mid}\right) \\
\% \text { Duplicados relativos WoS }=100 \times\left(\frac{\mid \text { Scopus } \cap \text { WoS } \mid}{\mid \text { WoS } \mid}\right)
\end{gathered}
$$

Respecto al análisis descriptivo-cualitativo, se estudió la evolución del número de publicaciones, qué fuentes concentraban un mayor número de trabajos en este ámbito, palabras clave más utilizadas y su evolución temporal y la adscripción de los investigadores en el tema que nos ocupa. Para ello, fue de gran ayuda el software VOS Viewer.

Además, para la evaluación del material (fase 5), esta metodología requiere clasificar las aportaciones seleccionadas, estableciendo dimensiones estructurales y categorías de análisis (véase tabla 1). Estas dimensiones se han seleccionado tomando como referencia estudios relacionados con revisiones bibliográficas vinculadas con la ecopedagogía y con temas afines a ella. En un análisis preliminar, se barajaron diferentes dimensiones estructurales y categorías de análisis, y finalmente, se aplicaron las que resultaban más consistentes con la clasificación del material encontrado relacionado con la ecopedagogía.

Tabla 1. Dimensiones estructurales y categorías de análisis

\begin{tabular}{|l|l|l|}
\hline $\begin{array}{c}\text { Dimensiones } \\
\text { estructurales }\end{array}$ & Categorías de análisis & \multicolumn{1}{|c|}{ Descripción de la categoría de análisis } \\
\hline \multirow{4}{*}{$\begin{array}{l}\text { Metodología de } \\
\text { investigación }\end{array}$} & Caso de estudio & $\begin{array}{l}\text { La metodología de investigación se centra en } \\
\text { analizar un caso práctico }\end{array}$ \\
\cline { 2 - 3 } & Revisión bibliográfica & $\begin{array}{l}\text { Estudios que analizan la literatura previamente } \\
\text { publicada }\end{array}$ \\
\cline { 2 - 3 } Tipo de & Teórico y conceptual & $\begin{array}{l}\text { El estudio desarrolla instrumentos teóricos } \\
\text { o empíricos o incluso aproximaciones para } \\
\text { evaluar y desarrollar la ecopedagogía }\end{array}$ \\
\hline \multirow{2}{*}{ investigación } & Marco teórico & $\begin{array}{l}\text { Realiza aportaciones teóricas al ámbito de la } \\
\text { ecopedagogía }\end{array}$ \\
\cline { 2 - 3 } & Análisis de experiencias & $\begin{array}{l}\text { Reflejan la experiencia vivida por } \\
\text { determinados colectivos tras desarrollar } \\
\text { actividades con metodologías ecopedagógicas }\end{array}$ \\
\cline { 2 - 3 } & Modelización & $\begin{array}{l}\text { Se desarrollan modelos estadísticos } \\
\text { relacionados con la ecopedagogía }\end{array}$ \\
\cline { 2 - 3 } & Encuestas & $\begin{array}{l}\text { La investigación ha desarrollado encuestas } \\
\text { para después analizar el tema que nos ocupa }\end{array}$ \\
\hline
\end{tabular}




\begin{tabular}{|l|l|l|}
\hline $\begin{array}{l}\text { Tipo de } \\
\text { investigación }\end{array}$ & Políticas de educación & $\begin{array}{l}\text { Analizan el impacto de una política educativa } \\
\text { existente o potencial para el desarrollo de la } \\
\text { ecopedagogía }\end{array}$ \\
\hline \multirow{5}{*}{ Nivel de análisis } & Macro & $\begin{array}{l}\text { Analizan la ecopedagogía y aspectos } \\
\text { relacionados con ella como un todo (por } \\
\text { ejemplo, actividades relacionadas con la } \\
\text { ecopedagogía en un territorio, así como } \\
\text { los estudios que desarrollan metodologías } \\
\text { orientadas a instaurar en el sistema educativo } \\
\text { la ecopedagogía) }\end{array}$ \\
\cline { 2 - 3 } & Meso & $\begin{array}{l}\text { Recoge las investigaciones intermedias que } \\
\text { se encuentran entre el nivel macro y micro, } \\
\text { por ejemplo, los estudios que realizan análisis } \\
\text { comparando varios centros educativos }\end{array}$ \\
\cline { 2 - 3 } & Micro & $\begin{array}{l}\text { Recoge todas las investigaciones relacionadas } \\
\text { con el estudio de un caso concreto, como por } \\
\text { ejemplo las experiencias realizadas en un aula }\end{array}$ \\
\hline $\begin{array}{l}\text { Campo } \\
\text { semántico de las } \\
\text { palabras clave }\end{array}$ & Ecopedagogía & $\begin{array}{l}\text { Las publicaciones consideradas se han } \\
\text { clasificado atendiendo a la temática (y por } \\
\text { ende, al campo semántico) que analizan }\end{array}$ \\
\cline { 2 - 3 } & Educación ambiental \\
\cline { 2 - 3 } & Sostenibilidad &
\end{tabular}

\section{Resultados}

La búsqueda de las aportaciones de ecopedagogía en las bases de datos WoS y Scopus generó un resultado total de 93 referencias (58 de WoS y 35 de Scopus). Sin embargo, se registraron 27 casos duplicados, por lo que finalmente contamos con 66 trabajos. Resulta llamativo el bajo número de publicaciones relacionadas con el tema de estudio, a pesar de la importancia de metodologías para estimular la empatía hacia el medio ambiente, así como valores relacionados con la justicia social o la equidad. Pese a ello, hay que resaltar que el número de documentos relacionados con la ecopedagogía en ambas bases de datos es relativamente bajo.

Figura 2. Proceso de selección del material bibliográfico

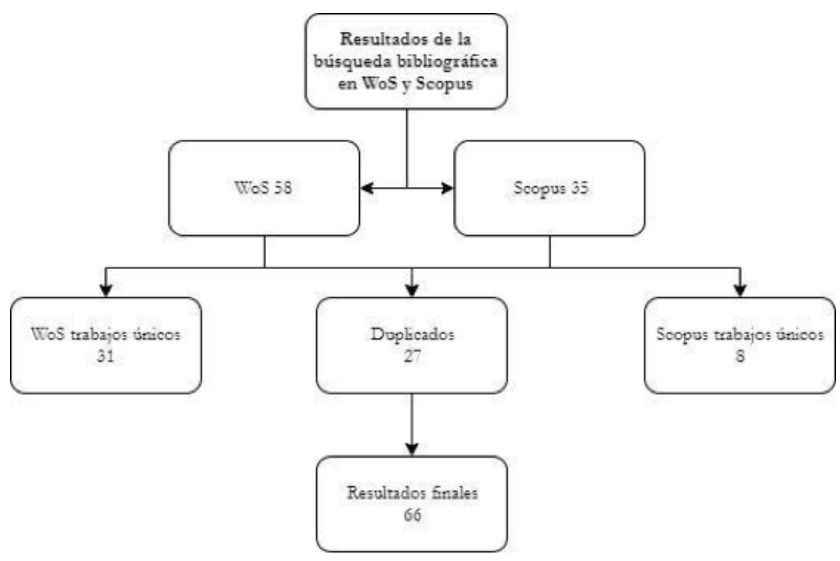




\section{Análisis descriptivo-cuantitativo. Exhaustividad de las bases de datos consultadas en materia de ecopedagogía}

La tabla 2 muestra los resultados obtenidos tras aplicar los distintos índices a WoS y Scopus, demostrándose que, en el ámbito de la ecopedagogía, WoS es la base de datos que mejor abarca el tema.

Tabla 2. Resultados de los índices para WoS y Scopus

\begin{tabular}{|l|c|l|}
\hline \multicolumn{1}{|c|}{ Índice } & $\begin{array}{c}\text { Resultados } \\
\text { obtenidos }\end{array}$ & \multicolumn{1}{c|}{ Interpretación } \\
\hline Índice Meyer Scopus & $59,70 \%$ & $\begin{array}{l}\text { WoS tiene una mayor cobertura de trabajos } \\
\text { relacionados con la ecopedagogía. }\end{array}$ \\
\hline Índice Meyer WoS & $67,40 \%$ & $\begin{array}{l}\text { El 40,91\% del material sobre ecopedagogía se } \\
\text { encuentra identificado en ambas bases de datos. } \\
\text { Es decir, buscando en una única base de datos, se } \\
\text { podrían identificar el } 59,09 \% \text { (100\%-40,91\%) de las } \\
\text { publicaciones únicas o que no están duplicadas. }\end{array}$ \\
\hline $\begin{array}{l}\text { Índice de } \\
\text { Solapamiento }\end{array}$ & $40,91 \%$ & $\begin{array}{l}\text { WoS abarca más material sobre ecopedagogía } \\
\text { que Scopus, con un } 77,14 \%, \text { mientras que Scopus } \\
\text { representa el 46,51\% de WoS. }\end{array}$ \\
\hline $\begin{array}{l}\text { \% Duplicados } \\
\text { relativos Scopus }\end{array}$ & $77,14 \%$ Duplicados \\
relativos WoS
\end{tabular}

Análisis descriptivo-cualitativo. Distribución y evolución del material bibliográfico a lo largo del tiempo, fuentes y autores

Ambas bases de datos muestran que la ecopedagogía no es un movimiento nuevo, sino que se remonta a las aportaciones de Freire en la década de los 70 del pasado siglo, pero no es hasta finales del siglo XX cuando empieza a crecer el número de publicaciones sobre ecopedagogía. De hecho, el $60 \%$ del material recopilado se ha escrito en los últimos cinco años.

El mayor número de investigaciones relacionadas con la ecopedagogía se han publicado en International Journal of Environmental and Science Education, en Praxis-Colombia y en ETD Educaçao Tematica Digital, seguidas muy de lejos por British Journal of Sociology of Education, Geographical Journal and European Journal of Education. En cuanto a los autores más prolíficos en ecopedagogía, hay que destacar Payne (Australia) con 7 publicaciones, Misiaszek (Estados Unidos) con 6 investigaciones, seguidos de Nakagawa (Australia), Gadotti (Brasil) y Magalhaes (Brasil).

\section{Análisis de las dimensiones estructurales y categorías de análisis}

Tras clasificar el material bibliográfico según las dimensiones y categorías de análisis definidas (véase tabla 1), se llevó a cabo un análisis pormenorizado de cada una de ellas.

La dimensión estructural metodología de investigación fue extraída de estudios vinculados con la sostenibilidad ambiental (Seuring y Müller, 2008). En nuestro 
caso, de las 66 referencias el 71,2\% son de tipo teórico y conceptual, seguidas de los casos de estudio con un 24,2\% y finalmente las revisiones bibliográficas, con el $4,6 \%$ restante.

Respecto a la dimensión estructural tipo de investigación, la mayoría de las publicaciones consideradas $(71,3 \%)$ se incluyen en la categoría de marco teórico, seguida del análisis de experiencias con un 13,7\% de los estudios totales. Los análisis estadísticos relacionados con la ecopedagogía representan un $9 \%$ y, finalmente, las publicaciones que realizan encuestas o que analizan políticas de educación suponen un $3 \%$ cada una de ellas.

La dimensión estructural nivel de análisis hace referencia al ámbito del estudio: macro, meso y micro. La mayoría de los estudios considerados pertenecen al nivel macro, de carácter genérico, y realizan estudios relacionados con la ecopedagogía para el conjunto de la sociedad, o analizan la transversalidad de este movimiento. El nivel macro concentra el $72,7 \%$ de las investigaciones, seguido del micro (casos concretos, experiencias, etc.) con un 19,7\% y el nivel meso, un nivel intermedio entre el macro y el micro, con el restante $7,6 \%$.

La última dimensión estructural es el campo semántico de las palabras clave de las investigaciones consideradas. En este caso, se han realizado dos análisis. Por un lado, se han establecido tres campos semánticos tras analizar las palabras clave de los 66 estudios seleccionados: sostenibilidad, educación ambiental y ecopedagogía.

Lógicamente, dado que la búsqueda en ambas bases de datos se estableció el término ecopedagogía, el 57,6\% de estas publicaciones emplean este concepto en sus palabras clave, seguidas de aquellas que están más enfocadas hacia la educación ambiental y la sostenibilidad con un $36,4 \%$ y un $6 \%$ respectivamente. Resulta necesario indicar que, las 66 investigaciones que se han seleccionado hacen referencia en mayor o menor grado la ecopedagogía, sin embargo, lo que aquí se analiza es el enfoque que realiza cada investigación.

Por otro lado, se ha estudiado la relación existente entre las palabras clave, para visualizar cuáles son utilizadas con mayor o menor intensidad, y las relaciones entre ellas en los documentos analizados. La figura 3 muestra círculos más grandes para las palabras clave más utilizadas. En este caso, ecopedagogy es el nexo común de los artículos considerados, palabra que ha sido aplicada por el 50\% de trabajos considerados, seguida muy de lejos de environmental education con un 6,06\%, seguidas de ecocriticism, environmental learning, education, environment, todas ellas en torno al $4,55 \%$.

Por su parte, la figura 4 muestra la evolución en el uso de esas palabras clave. Las palabras clave más antiguas, es decir, las que se llevan empleando desde 2005 (color más oscuro) son: ecopedagogy, political ecology o environmental learning, mientras que las más novedosas, aquellas que se han empleado en 2019 (color más claro) son: early childhood education, outdoor teaching and learning, nature-culture o transformative education. 
Figura 3. Interrelación entre las palabras clave utilizadas en los artículos seleccionados

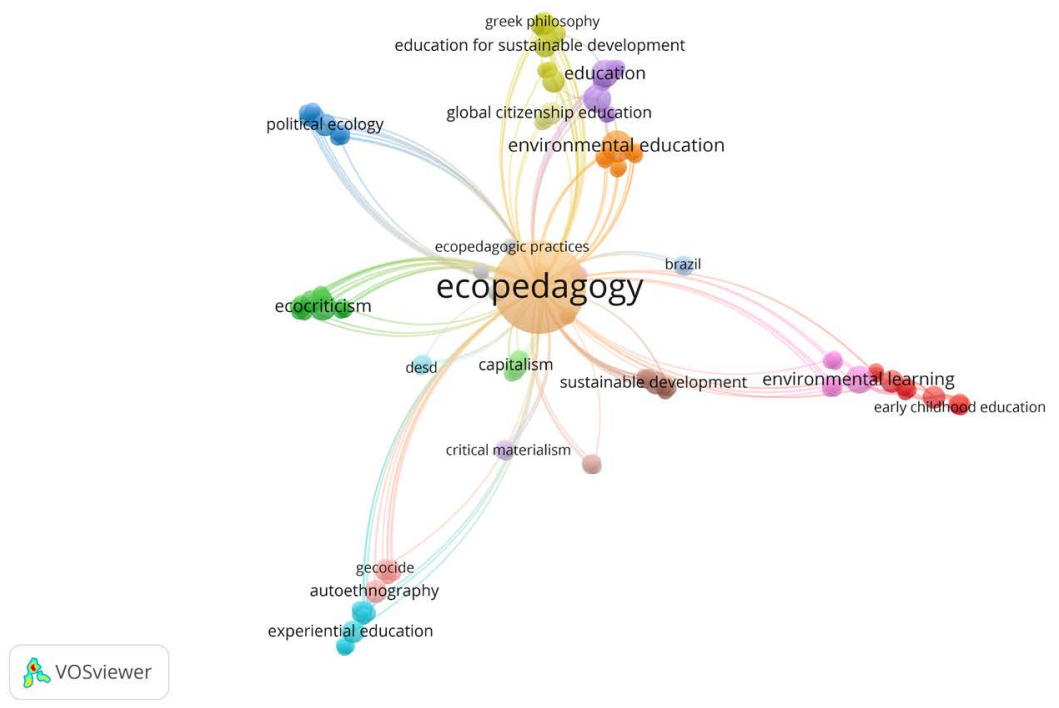

Figura 4. Evolución en el uso de las palabras clave. Periodo 2005-2019 (noviembre)

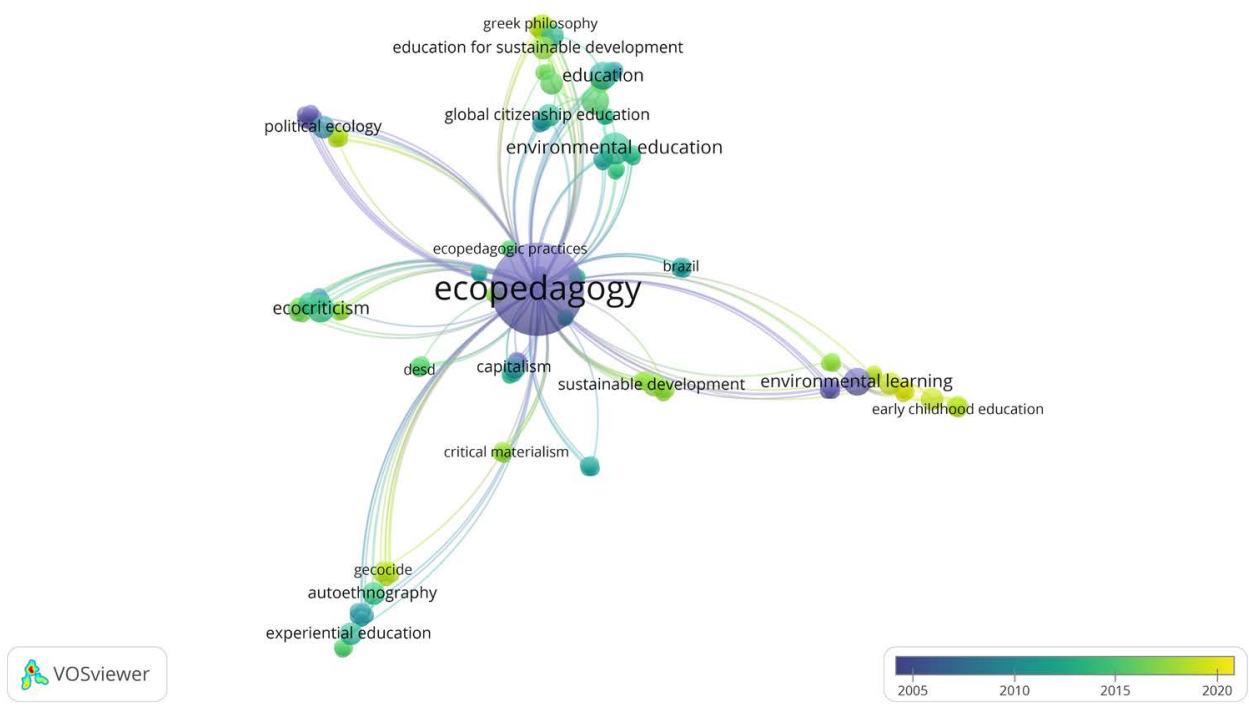

\section{Análisis del material bibliográfico}

Como se ha comentado, la ecopedagogía se conoce globalmente a raíz de la firma de la CT, entendiéndose como "un movimiento ético global para llegar a un código planetario de ética, sosteniendo un núcleo de principios y valores que hacen 
frente a la injusticia social y a la falta de equidad reinante en el planeta. Cinco cimientos sostienen ese núcleo: a) derechos humanos; b) democracia y participación; c) equidad; d) protección de la minoría; e) resolución pacífica de conflictos" (Gadotti, 2001: 68). En este sentido, la ecopedagogía o pedagogía de la Tierra surge como un movimiento que busca una pedagogía apropiada que se ajuste a los requerimientos de la CT y se extienda por todo el planeta, tratando de aplicar los principios de la CT en el currículo educativo, para implementar un cambio social a nivel global. De hecho, Gadotti (2004) propuso que la ecopedagogía supusiera una nueva visión de la educación a nivel global que se ajustase a las transformaciones globales y a las necesidades que presentaban los desafíos del nuevo siglo.

La ecopedagogía se llamó inicialmente, ecopedagogía del desarrollo sostenible, un término inadecuado, si se tiene en cuenta que la visión del neoliberalismo de desarrollo sostenible, es antiecológica y que en muchas ocasiones es utilizada por grandes empresas para aparentar ser verde cuando no se es (Fernández y Conde, 2010). En sus orígenes, el desarrollo sostenible (CMMAD, 1987) era contrario al crecimiento económico, ya que el desarrollo económico (y no, crecimiento económico) quedaba subordinado a la necesidad de garantizar un uso sostenible de los recursos naturales, la salud de los ecosistemas y la protección de la biodiversidad, presentándose este concepto como un obstáculo para la economía y entrando en conflicto con la doctrina del neoliberalismo, cuya hegemonía creció en la década de los 80, especialmente con la culminación de la Guerra Fría en 1989. De hecho, Margaret Tatcher y Ronald Reagan, afirmaban que el desarrollo sostenible cosificaba a la economía, argumentando que, tanto los medios como los fines del desarrollo económico cuestionaban el bienestar económico de los pueblos. Según Evans y Reid (2014), a pesar de que el desarrollo sostenible surgió como una crítica al neoliberalismo, las adaptaciones del original concepto de Brundtland al marco neoliberal del momento, hizo aún más poderosas las bases de los fundamentos neoliberales. Por ejemplo, Mark Duffield (en Evans y Reid, 2014) Ilama la atención en los países más pobres, donde se defendía que el desarrollo sostenible solo se alcanzaría practicando la autosuficiencia basada en la comunidad, por lo que el desarrollo sostenible reflejó una agenda política neoliberal que trasladaba la carga de la seguridad de los Estados a las personas, a quienes se les exige demostrar su valía mejorando su autosuficiencia individual y colectiva.

Según Martínez-Rodríguez, Vilches y Fernández-Herrería (2018) las instituciones públicas se han ido moldeando a las exigencias del capitalismo neoliberal para legitimarlo, siendo el sistema educativo el medio ideal para culminar la expansión global del neoliberalismo, ya que la educación cada vez más, se contempla como un negocio, perdiendo su tradicional rol de servicio público al ofrecerse como un bien de consumo en la sociedad. Un claro ejemplo de la estandarización de la educación es el caso de la educación superior en la Unión Europea. En efecto, tras la búsqueda de un sistema basado en competencias, busca homogeneizar los currículos universitarios bajo el pretexto de mejorar la empleabilidad de los estudiantes, ajustar sus estudios al mercado de trabajo, incrementar la producción empresarial y, en definitiva, estimular el crecimiento económico.

En este sentido, y en el ámbito de la educación, Huckle y Wals (2015) sostienen en su discurso, que las propuestas de la ONU al referirse al desarrollo sostenible, a la Agenda 2030, o cuando la UNESCO se refiere a la Década de la Educación para el 
Desarrollo Sostenible (DESD 2005-2014) son propuestas esencialmente reformistas con influencias neoliberales, ya que prestan muy poca atención al poder, la política y a la ciudadanía. Por ello, la EDS adaptada a las necesidades de estas propuestas, con claros matices neoliberales, no es suficiente para llevar a cabo la transformación en las relaciones humanas, sociales y ambientales vigentes, siendo este, el fundamento de la ecopedagogía suscrita por Gadotti (2001). Siguiendo a Selby y Kagawa (2010), la EDS cuenta con una definición poco rigurosa, que tiende a difuminarse y que pone al descubierto inconsistencias e incompatibilidades, que subrayan, una vez más, la importancia del crecimiento económico frente a las esferas social y ambiental.

En un intento de solventar esta brecha entre la EDS y la ecopedagogía, Huckle y Wals (2015) plantean lo que denominan Educación Global para la Ciudadanía Sostenible (Global Education For Sustainability Citizenship, GESC), describiendo cuatro dimensiones de dicha educación (dimensión a escala global, que pretende introducir a los estudiantes en una sociedad global, en el sentido de que las decisiones personales y colectivas tienen impactos en personas de otros territorios, así como en el resto de seres vivos; dimensión ética, consistente en que los estudiantes reconozcan la sostenibilidad como una noción normativa y cómo los principios de la CT podrían permitir el desarrollo de una sociedad basada en el respeto por la naturaleza, los derechos humanos universales, la justicia económica y una cultura de paz; la dimensión relacional enfocada en la construcción social de tales conceptos, como sostenibilidad y ciudadanía, y requiere que los estudiantes comprendan que si bien hay aceptación generalizada de los principios de la CT entre las organizaciones mundiales, la sostenibilidad y ciudadanía pueden basarse en otros valores e intereses comunes; y la dimensión política, centrada en cuestiones de justicia social y ambiental) para acercar la educación mercantilizada a los principios de la ecopedagogía. En línea con este pensamiento de transformación que ha quedado invisibilizado por el neoliberalismo, otros autores plantean que, mientras que el concepto de desarrollo sostenible puede ser voluble, los principios de la CT son difícilmente vulnerables, y son estos los que deben fundamentar la ecopedagogía y los currículos académicos. Así pues, Hargreaves (2003) planteó la necesidad de que las escuelas deben de estar interconectadas con las comunidades que las integran, y los currículos deben tener una mayor profundización en la inteligencia emocional de educandos y educadores. Este argumento se refuerza con los trabajos de Dobson (2003) y Lanz (2005) que mencionan que la ecopedagogía pretende educar ciudadanos miembros de una comunidad: la Tierra, y es por ello, por lo que se debe despertar el sentimiento de pertenencia al planeta Tierra y a todo lo que hay en él, conectándonos con todo lo que nos rodea, y formando un sistema sólido y común a nivel global. Otros autores aportan otras variantes como slow ecopedagogy o ecopedagogía del decrecimiento. Fernández y Conde (2010) y Mallart y Solaz (2008) indican que el mismo concepto desarrollo sostenible está en crisis, y que no es suficiente mantener el crecimiento cero, sino que además hay que disminuirlo. Justifican teorías de decrecimiento económico como única solución a los problemas ambientales actuales, un desarrollo sin crecimiento en el uso de recursos y en las cargas ambientales por encima de la capacidad de carga del ecosistema. El decrecimiento o slow economy también implica un cambio de modelo económico y social junto a la educación de los jóvenes y la re-educación de los adultos (Mallart y Solaz, 2008). A partir de estas teorías, 
conceptos como slow food, slow cities, slow travel, y cómo no, slow ecopedagogy o ecopedagogía del decrecimiento, tratan de instaurar principios como menos es más o menos es mejor en el ámbito educativo.

En general, si se quiere llevar a cabo una transformación en las relaciones humanas, sociales, económicas y ambientales, se deben considerar cambios significativos en los procesos productivos, en el uso de los recursos, en la actual organización económica internacional, en la gobernanza, el rol de la ciudadanía, etc., impuestos por un neoliberalismo que ha priorizado el beneficio individual frente al de la comunidad (Dobson, 2003).

A pesar de su carácter multidisciplinar y transversal, la ecopedagogía sigue teniendo poca visibilidad en cuanto a publicaciones de carácter científico y didáctico se refiere. Como era de esperar, la ecopedagogía ha tenido un especial desarrollo en Brasil, cuna del concepto, donde se han desarrollado diferentes proyectos de gran envergadura. Además de los programas específicos del Grado de Ecopedagogía y del Instituto Paulo Freire (al mando de Gadotti), que fomentan este movimiento, hay muchos casos de ecopedagogía que no sólo enseñan a las personas el pensamiento crítico en esta disciplina, sino que las involucran en el aprendizaje a través de la acción, de la experiencia. Este es el caso de De Sousa et al. (2015), quienes utilizaron prácticas ecopedagógicas para el proceso de inclusión escolar de niños con discapacidad en tres centros educativos de Bahía (Brasil), demostrándose que la ecopedagogía en proyectos ambientales en las aulas, favorecen la inclusión, eliminándose barreras físicas o de comunicación. También existen casos prácticos en el entorno universitario. Eryaman et al. (2010) desarrollaron un programa de ecopedagogía para profesores universitarios, estudiantes y los padres de estos, donde se trataron cuestiones ambientales, sociales y económicas locales, lo que hizo que los participantes estén más motivados e implicados en realizar aportaciones en un territorio que conocen y en el que tienen recuerdos y experiencias. Otro ejemplo, es el desarrollado por Tinnell (2011), quien desarrolló el proyecto ecoblogging, basado en ideas y prácticas de justicia ambiental y sostenibilidad para garantizar una mejor calidad de vida. Al difuminar los límites entre la naturaleza y la cultura, el proceso de ecoblogging empuja a los estudiantes a reflexionar no solo sobre las amenazas ambientales específicas, sino también sobre los valores y prácticas culturales que amenazan, marginan y fragmentan las ecologías sociales particulares. Otros estudios aplican la ecopedagogía al turismo concienciando al visitante de la importancia de preservar el entorno natural, la cultura, los valores y las tradiciones del lugar de destino (Nakagawa, 2018).

De igual modo, otras muchas prácticas no suponen cambios significativos en el sentido de la ecopedagogía, sino que se trata de actuaciones superficiales, que en un principio pueden parecer críticas y transformadoras, pero que finalmente, son llevadas a cabo por el propio sistema, anulando su potencial de cambio. Esta es la conclusión de LæssØe (2010), que, a través de tres casos prácticos en un entorno educativo informal, demostró que, a pesar de que se proporciona un marco relativamente abierto para estimular prácticas innovadoras, existen ciertas cuestiones que tienden a mantenerse al margen en el discurso dominante del manipulado desarrollo sostenible.

En esta revisión bibliográfica también se han encontrado numerosas prácticas en distintos niveles educativos que no profundizan en las transformaciones de la ecopeda- 
gogía basada en la CT. Este es el caso de Capra y Luisi (2014) que defienden una programación académica, en la que los educadores establezcan los contenidos ambientales según su experiencia personal; McNaughton (2010) presenta un estudio realizado con niños de 10 años, concluyendo que la educación a través de representaciones teatrales tiene éxito al involucrar a los estudiantes en las dimensiones ecológicas, sociales y políticas de problemas globales como los residuos sólidos y la deforestación. Payne (2010) y Rainbow (2012) destacan la implantación de la ecopedagogía en el mundo de la imaginación a través de historias y cuentos, lo denominan ecoliteracy puesto que la literatura permite y favorece la sensibilización, abriendo una importante dimensión experiencial de tomar conciencia ecológica y respeto hacia la naturaleza.

Asimismo, hay autores que cuestionan la ecopedagogía. Bowers $(2005,2012)$, criticado por Freire, argumenta que, si la ecopedagogía se adoptara globalmente, contribuiría a la difusión hegemónica de la cultura y el pensamiento occidental, dejando al margen otras formas no occidentales de pensar, desarrollando un monocultivo mundial. Basándose en los argumentos de Bowers, otros autores indican que la ecopedagogía en sí es útil, pero añaden que carece de fundamento y sería contraproducente en sus objetivos, sin un enfoque constante en la compresión y en el fomento de la diversidad de pensamiento, en la cultura y en el ecosistema (Gruenewald, 2005). Se puede decir que se requieren diferentes ecopedagogías para distintos pensamientos, culturas y entornos naturales.

\section{Conclusiones}

El interés de una revisión de las aportaciones que se han hecho hasta ahora sobre el tema de la ecopedagogía ha ido encaminado a conocer su nivel de implantación en el contexto educativo formal e informal como proceso de aprendizaje para la conformación de una concienciación de justicia ambiental y social, más sólida, desarrollando el sentido de pertenencia a una comunidad.

El análisis bibliométrico identificó a WoS como la base de datos que mejor recoge las aportaciones realizadas sobre ecopedagogía y presenta óptimos resultados en duplicidad y solapamiento frente a Scopus. Asimismo, este análisis manifiesta que, si bien la ecopedagogía es una metodología docente con un desarrollo epistemológico que abarca 4 décadas, no es hasta 2010 cuando se difunden la mayoría de aplicaciones, sobre todo desde países anglosajones y Sudamérica.

En relación al ámbito y a los límites de actuación de la ecopedagogía, se ha observado una importante dispersión de temas y enfoques, motivada por la diversidad profesional de quienes han tratado el tema. En general, la mayoría de los estudios tienen un marcado carácter teórico, y muchos de ellos son críticos con los actuales contextos educativos, poniendo de manifiesto la necesidad de un cambio. Asimismo, existen numerosos documentos que muestran experiencias prácticas relacionadas con la ecopedagogía, pero la mayoría no profundiza en los problemas que esta pedagogía pretende solventar.

Tras la revisión realizada, se pone de manifiesto que no se han tratado los problemas en las aulas como se debía, no haciendo partícipes a los estudiantes en una cuestión tan crucial como es la protección ambiental, la equidad o la justicia social. 
Además, encontramos numerosos autores críticos con la ecopedagogía por carecer de una base sólida de fundamentos, por cómo se está enfocando o por su metodología pedagógica, denotando carencias en cuanto a un enfoque global que considere la diversidad de pensamiento, culturas y entornos naturales. Algunos ejemplos como el slow ecopedagogy han intentado solventar el enfoque actual de la ecopedagogía para orientarla hacia las teorías del decrecimiento económico y acercar el cambio de modelo económico y social en el ámbito educativo.

Sin embargo, en cualquiera de las variantes de la ecopedagogía, la finalidad común es desarrollar una conciencia ambiental crítica y comprometida que sea sostenible con las esferas social y económica. Sin embargo, y muchos autores coinciden en ello, no es posible desarrollarla en un contexto neoliberal, en el que esa conciencia se desarrolla desde una silla (a modo individual) dentro del aula. Es aquí donde se encuentra otro de los principales retos de la educación del siglo XXI. En este sentido, la ecopedagogía requiere vivir experiencias que nos reconecten de nuevo con la naturaleza y con el territorio, haciendo hincapié tanto en la relación existente entre medio social, el medio natural y económico como en experiencias más cercanas al espacio vital del estudiante.

Existen numerosas prácticas, que aunque pretenden aplicar este planteamiento, no implementan las transformaciones necesarias que demanda la ecopedagogía. Siguiendo a Jickling y Wals (2007) existe una clara contraposición entre el originario concepto de desarrollo sostenible y el actual enfoque de la educación ambiental. Según estos autores, la EDS es un producto más del proceso de globalización del neoliberalismo, y supone una serie de arreglos transmisivos para enseñar y aprender más que transformadores. En este contexto, defienden la heurística como una herramienta crítica que se puede emplear para hacer crítica del discurso neoliberal actual, evaluar nuevas iniciativas, y encontrar el propio lugar en los debates actuales, pero también es necesario para apoyar el inconformismo, y aportar iniciativas educativas sobre pobreza, salud, justicia social, desarrollo y otras agendas globales. Una heurística que recupere la justicia y desarrollo sostenible, es decir, recuperar el original concepto de desarrollo sostenible y rectificar injusticias, implementando la justicia intergeneracional, la justicia social y la justicia global, tal como indicaba Brundtland, sin olvidar la justicia en las relaciones humanas con los animales y el entorno natural (Barry, 2005).

En nuestra opinión, y tras haber realizado esta revisión bibliográfica, subrayamos la necesidad imperante de introducir cambios en el sistema educativo, que incluyan los principios originarios de la ecopedagogía, tal y como se recoge en la CT o en los trabajos de Freire. A día de hoy, existen importantes problemas en las esferas social, ambiental y económica, que son susceptibles de reconducirse a través de una población instruida y consciente de la importancia de transformar y mantener un equilibrio entre esos tres ámbitos, y la ecopedagogía se plantea como la opción ganadora. Asimismo, consideramos de crucial importancia, que no se puede desarrollar una única ecopedagogía global, sino que debe de instaurarse una ecopedagogía libre de matices neoliberales y adaptada a las circunstancias de cada territorio, de cada comunidad, reflexionando sobre cómo se desarrollan las relaciones sociales, culturales, éticas, de género, ambientales, económicas, etc., que nos resultan afines, para así poder educar un capital humano capaz de realizar críticas constructivas del entorno que conoce, que le es familiar. 
En términos generales, los actuales contextos educativos deben de adaptarse a las circunstancias cambiantes y deben producirse cambios importantes que aíslen a la educación de la mercantilización a la que se ve sometida, y alejarse de matices neoliberales si realmente pretendemos reconducir las actuales tendencias sociales, económicas y ambientales que nos llevan a la deriva.

\section{RefERENCIAS BIBLIOGRÁFICAS}

Aghaei, A., Salehi, H., Yunus, M. M., Farhadi, H., Fooladi, M., Farhardi, M. y Ebrahim N. A. (2013). A comparison between two main academic literature collections: Web of science and Scopus databases. Asian Social Science, 9(5), 18-26. https:// doi.org/10.5539/ass.v9n5p18

Antunes, A. y Gadotti, M. (2006). La ecopedagogía como la pedagogía indicada para el proceso de la Carta de la Tierra. En P. B. Corcoran (Ed.), La Carta de la Tierra en Acción. Hacia un mundo sostenible (pp. 141-143). Amsterdam: Kit Publishers.

Barry, J. (2005). Resistance is Fertile: From Environmental to Sustainability Citizenship. En D. Bell y A. Dobson (Eds.), Environmental Citizenship: Getting from Here to There? (pp. 21-48). Shandong: MIT Press.

Bowers, C. A. (2005). How Peter McLaren and Donna Houston, and Other "Green" Marxists Contribute to the Globalization of the West's Industrial Culture. Educational Studies, 37(2), 185-195. https://doi.org/10.1207/s15326993es3702_8

Bowers, C. A. (2012). Questioning the idea of the individual as an autonomous moral agent. Journal of Moral Education, 41(3), 301-310. https://doi.org/10.1080/03057 240.2012.691626

Capra, F. y Luisi, P. L. (2014). The Systems View of Life: A Unifying Vision. Cambridge: Cambridge University Press.

CMMAD, Comisión Mundial sobre Medio Ambiente y Desarrollo. (1987). Report of the World Commission on environment and Development: Our Common future (Informe Brundtland). Paper presented at the Development and International EConomic Co-Operation: Environment (A745/427).

Denyer, D. y Tranfield, D. (2009). Producing a systematic review. En A. Buchanan, A. Bryman (Eds.), SAGE Handbook of Organizational Research Methods (pp. 671689). London: SAGE.

De Sousa, N. A., da Silva, M. F. y Costa, S. K. (2015). A ecopedagogia como prática ecopedagógica inclusiva em escolas de Vitória da Conquista na Bahia. REMEARevista Electrónica do Mestrado em Educaçao Ambiental, 32(1), 247-269. https:// periodicos.furg.br/remea/article/view/5072

Dobson, A. (2003). Citizenship and the Environment. Oxford: OUP.

Durán, A., del Río, M. C. y Álvarez, J. (2016). Bibliometric analysis of publications on wine tourism in the databases Scopus and WoS. European Research on Management and Business Economics, 23(1), 8-15. https://doi.org/10.1016/j. iedeen.2016.02.001

Eryaman, M. Y., Yalcin-Ozdilek, S., Okur, E., Cetinkaya, Z. y Uygun, S. (2010). A Participatory Action Research Study of Nature Education in Nature: Towards Community-based Eco-pedagogy. International Journal of Progressive Education, 6(3), 26-37. https://eric.ed.gov/?id=EJ919474 
Evans, B. y Reid, J. (2014). Resilient life: The art of living dangerously. Cambridge: Polity.

Fernández, A. y Conde, J. L. (2010). La ecopedagogía en la formación inicial de maestros. Investigación en la Escuela, 71, 39-49. http://doi.org/10.12795/IE.2010. i71.04

Freire, P. (1993). Pedagogía de la esperanza: un reencuentro con la pedagogía del oprimido. México D.C.: Siglo Veintiuno.

Gadotti, M. (2006). La pedagogía de Paulo Freire y el proceso de democratización en el Brasil: algunos aspectos de su teoría, de su método y de su praxis. En A. Ayuste (Coord.), Educación, ciudadanía y democracia (pp. 39-64). Barcelona: Octaedro.

Gadotti, M. (2004). Os Mestres de Rousseau. Sâo Paolo: Cortez.

Gadotti, M. (2001). Pedagogía de la Tierra y cultura de la sustentabilidad. Revista Paulo Freire. Revista de pedagogía crítica, 2(2), 61-76. https://doi. org/10.25074/07195532.2.519

Gadotti, M. (2000). Pedagogía da Terra. Sâo Paolo: Peirópolis.

Gluck, M. (1999). A review of journal coverage overlap with an extension to the definition of overlap. Journal of the Association for Information Science and Technology, 41(1), 43-60. https://doi.org/10.1002/(SICI)1097-4571(199001)41:1<43::AID$\mathrm{ASI} 4>3.0 . \mathrm{CO} ; 2-\mathrm{P}$

Gruenewald, D. A. (2005). More than one profound truth: Making sense of divergent criticalities. Educational Studies, 37(2), 206-214. https://doi.org/10.1207/ s15326993es3702_10

Gutiérrez, F. y Prado C. (2000). Ecopedagogía y ciudadanía planetaria. Valencia: Diálogos.

Hargreaves, A. (Comp.) (2003). Replantear el cambio educativo. Un enfoque renovador. Madrid: Amorrortu.

Huckle, J. y Wals, A. E. J. (2015). The UN decade of education for sustainable development: business as usual in the end. Environmental Education Research, 21(3), 491-505. https://doi.org/10.1080/13504622.2015.1011084

IUCN, International Union for Conservation of Nature and Natural Resources (1980). Word Conservation Strategy. Switzerland: Morges.

Jickling, B. y Wals, A. (2007). Globalization and environmental education: Looking beyond sustainable development. Journal of Curriculum Studies, 40(1), 1-21. https://doi.org/10.1080/00220270701684667

Jiménez, R. y García, E. (2017). Visibilidad de la Educación Ambiental y de la Educación para la Sostenibilidad en las publicaciones españolas sobre educación científica. Revista Eureka sobre Enseñanza y Divulgación de las Ciencias, 14(1), 271 285. https://doi.org/10.25267/Rev_Eureka_ensen_divulg_cienc.2015.v12.i3.11

Kitcharoen, K. (2004). The importance-performance analysis of service quality in administrative departments of private universities in Thailand. ABAC Journal, 24, 20-46.

LæssØe, J. (2010). Education for sustainable development, participation and sociocultural change. Environmental Education Research, 16(1), 39-57. https://doi. org/10.1080/13504620903504016 
Lanz, S. (2005). Ecopedagogía y cultura depredadora. Revista Cubana de Educación Superior, 25(2), 59-70.

Mallart, J. y Solaz, C. (2008). Ecopedagogía del decrecimiento para la formación de una ciudadanía planetaria. Trabajo presentado en el XIV Congreso Nacional y III Iberoamericano de Pedagogía: "Educación, ciudadanía y convivencia", Zaragoza, España.

Magalhaes, H. G. D. (2013). A ecopedagogía e a pedagogía da informalidade na escola. REMEA-Revista Electrónica do Mestrado em Educaçao Ambiental, 30(2), 304-316. https://periodicos.furg.br/remea/article/view/3694

Martínez-Rodríguez, F. M., Vilches, M. A. y Fernández-Herrería, A. (2018). Challenging the neoliberal view of education: the Center for Ecoliteracy as a transformative educational practice. Globalizations, 432-436. https://doi.org/10.1080/14747 731.2018 .1446601

Matthews, J. (2014). Hybrid Pedagogies for Sustainability Education. The Review of Education, Pedagogy, and Cultural Studies, 33, 260-277. https://doi.org/10.1080/ 10714413.2011 .585288

McNaughton, M. J. (2010). Educational drama in education for sustainable development: ecopedagogy in action. Pedagogy, Culture \& Society, 18(3), 289-308. https://doi.org/10.1080/14681366.2010.505460

Nakagawa, Y. (2018). EscapeScape: Simulating ecopedagogy for the tourist. The Journal of Environmental Education, 49(2), 164-176. https://doi.org/10.1080/009589 64.2017.1417221

ONU (2015). Transformar nuestro mundo: Agenda 2030 para el Desarrollo Sostenible. (Informe No. A/RES/70/1). https://unctad.org/meetings/es/SessionalDocuments/ares70d1_es.pdf

Payne, P. G. (2010). Moral spaces, the struggle for an intergenerational environmental ethics and the social ecology of families: an "other" form of environmental education. Environmental Education Research, 16(2), 29-231. https://doi. org/10.1080/13504620903580545

Rainbow, A. (2012). Ecoliteracy, enchantment and consilience. Bucarest: Literacy and Cultural Studies Series.

Rodrigues, C. (2018). Movement-Scapes as ecomotricity in ecopedagogy. Journal of Environmental Education, 49(2), 88-102. https://doi.org/10.1080/00958964.2017 .1417222

Ruiz, S. M., Porcel, L. y Ruiz, A. I. (2018). El papel de la educación para introducir valores ambientales en la sociedad. En J. C. Martínez Coll (Coord.), III Congreso Internacional virtual sobre la educación en el siglo XXI (pp. 293-304). https://www. eumed.net/actas/18/educacion/24-el-papel-de-la-educacion.pdf

Selby, D. y Kagawa, F. (2010). Runaway climate change as challenge to the closing circle of Education for Sustainable Development. Journal of Education for Sustainable Development, 4(1), 37-50. https://doi.org/10.1177/097340820900400111

Seuring, S. y Müller M. (2008). From a literature review to a conceptual framework for sustainable supply chain management. Journal of Cleaner Production, 16, 16991710. https://doi.org/10.1016/j.jclepro.2008.04.020 
Tamarit, A. y Sánchez, C. (2004). Ecopedagogía en acción: Propuesta lúdica a partir de la "Carta de la Tierra". En A. Fernández (Presidencia), IV Congreso Estatal del Educador Social. Políticas socioeducativas: Retos y propuestas en el siglo XXI. Santiago de Compostela, España.

Tinnell, J. (2011). Scripting just sustainability: through green listing towards eco-blogging. Environmental communication, 5(2), 228-242. https://doi.org/10.1080/1752 4032.2011.562519

UNESCO, 2017. Educación para los Objetivos de Desarrollo Sostenible. (Informe No. 50982). Paris: UNESCO. https://unesdoc.unesco.org/ark:/48223/pf0000252423

WCED, World Commission on Environment and Development (1987). From One Earth to One World: An Overview. Oxford: Oxford University Press.

Whiting, K., Konstantakos, L., Misiaszek, G., Simpson, E. y Carmona, L. G. (2018). Education for the sustainable global citizen: what can we learn from stoic philosophy and Freirean environmental pedagogies? Education Sciences, 8, 204-218. https://doi.org/10.3390/educsci8040204 